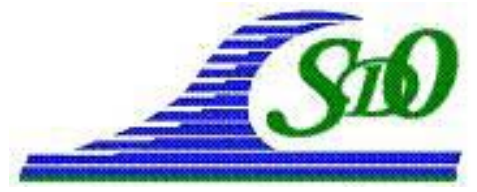

XI ${ }^{\text {èmes }}$ Journées Nationales Génie Côtier - Génie Civil

Les Sables d'Olonne, 22-25 juin 2010

DOI:10.5150/jngcgc.2010.077-D C Editions Paralia CFL

disponible en ligne - http://www.paralia.fr - available online

\title{
Elastocoast ${ }^{\circledR}$ - Process innovateur dans la construction d'ouvrages en enrochements dans le cadre de la protection du littoral maritime et fluvial
}

\author{
Philippe DE GARDER ${ }^{1}$
}

1. BASF Polyuréthanes France S.A.S., Z.I. de Mitry-Compans, Rue Decauville, BP 207, 77292 Mitry-Mory Cedex, France.

philippe.degarder@basf.com

\section{Résumé :}

Depuis 2004, le comportement structurel et écologique de PBA-REVETMENT ou agrégats liés par du polyuréthane mis en œuvre en Allemagne, en Hollande, en Angleterre et en France a été étudié. Les résultats en laboratoires et les tests sur prototypes montrent que ce nouveau matériau est prometteur de par ses propriétés techniques, son comportement mécanique et environnemental. La combinaison de ces trois caractéristiques, à savoir forte résistance, aspect naturel, compatibilité avec l'environnement, en fait une alternative sérieuse et attractive.

Les recherches sur les propriétés de ce matériau et sur les mécanismes de défaillance ont été réalisées en coopération entre BASF Polyurethanes $\mathrm{GmbH}$ pour ses compétences chimiques et les universités pour leurs compétences hydrauliques.

\section{Mots-clés :}

Polyuréthane - Protection du littoral - Agrégats - Structure poreuse - Absorption de l'énergie des vagues - Résistance - Aspect naturel - Environnement - Jet de rive

\section{Introduction}

Le polyuréthane est un nouvel agent liant pour les revêtements dans le secteur de l'ingénierie côtière. Il ouvre un large éventail d'applications et de possibilités. Le PBAREVETMENT (Polyurethan Bonded Aggregate Revetment) ou agrégats liés par du polyuréthane est un revêtement plat constitué d'agrégats liés ensemble par une résine polyuréthane. Chaque agrégat est individuellement collé par ses points de contact aux autres, d'où la structure poreuse de l'ouvrage.

\section{Elastocoast ${ }^{\circledR}$ : Le concept}

Pour mettre au point une nouvelle technique d'enrochements, nos équipes se sont heurtées à des contraintes environnementales majeures: résistance aux contraintes hydrauliques, stabilité dans le temps, neutralité en matière d'écotoxicité et intégration du matériau dans l'environnement naturel. 
Les enrochements conventionnels jointoyés présentent une porosité faible ou quasi nulle due à la proportion importante de matériau liant. Sur le long terme ces structures sont endommagées par l'impact des vagues et perdent leur efficacité, voir figure 1.

C'est pourquoi la technologie Elastocoast@ a été conçue pour lier les agrégats entre elles avec un fort coefficient de vide. On obtient ainsi un ouvrage poreux, tridimensionnel, monolithique et stable dont la caractéristique majeure est d'absorber l'énergie des vagues et par conséquent de mieux la dissiper, voir figure 1. Le résultat est une réduction de la hauteur de vague et une diminution de leur potentiel de détérioration.

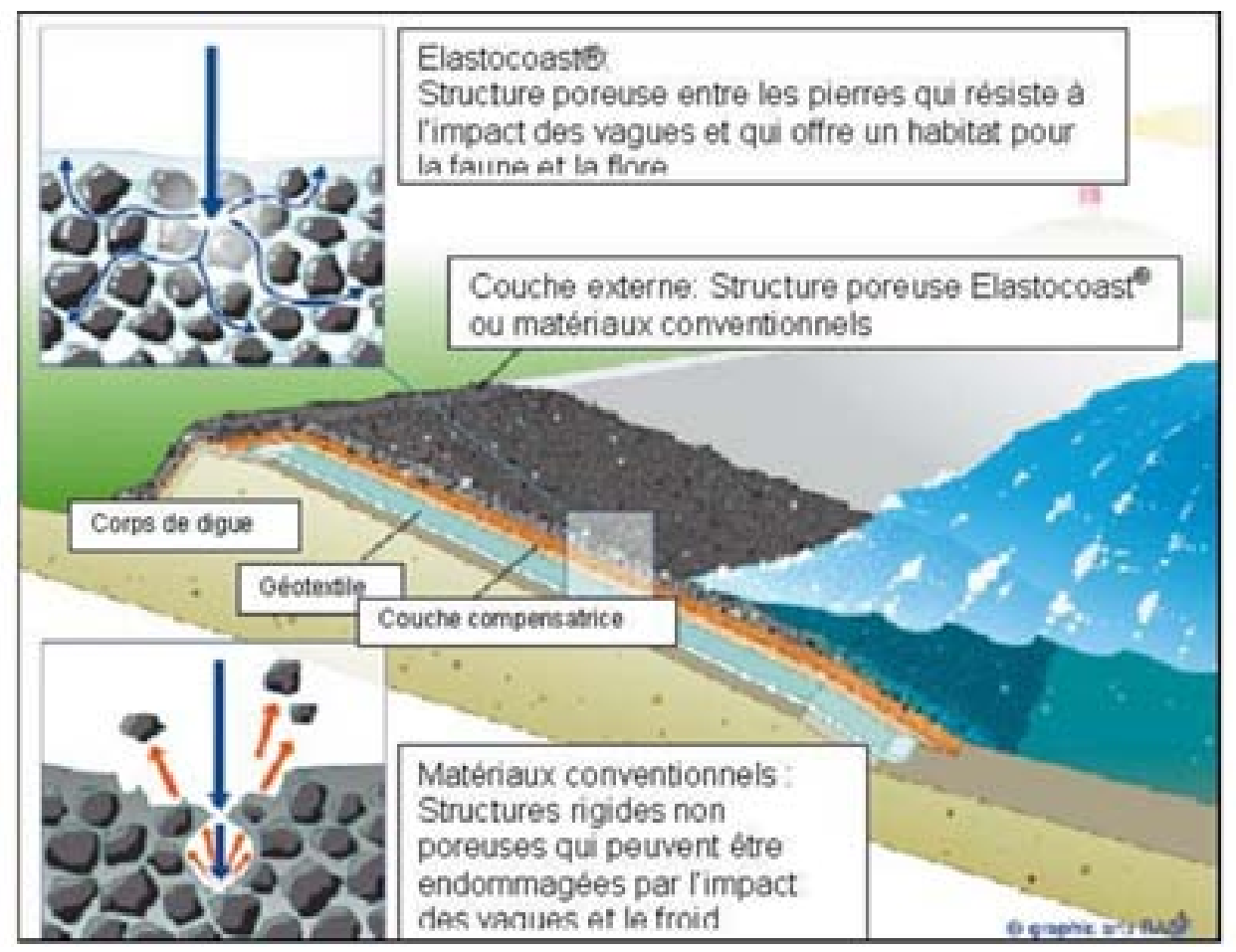

Figure 1. Exemple de construction d'un ouvrage en enrochements avec Elastocoast $\AA$.

\section{Procédé et mise en ouvre}

Ce procédé est très simple et réalisable sur place, voir figure 2. Dans une bétonnière classique, des agrégats de dimension réduite sont mélangés à froid avec environ $3 \%$ du volume correspondant d'Elastocoast ${ }^{\circledR}$ pendant 2 minutes. L'on dispose ensuite de 20 à 60 minutes pour répandre le mélange sur la surface à protéger. Le revêtement ainsi fabriqué peut être mis en charge après une journée.

Les enrochements doivent être propres et secs au toucher, condition facilement réalisable grâce à des mesures simples de manutention et de logistique.

Avant que la résine polyuréthane ne soit totalement sèche au toucher pour éviter de glisser et pour donner un aspect naturel à l'ouvrage, il est conseillé de projeter du sable sec sur la surface du revêtement. 

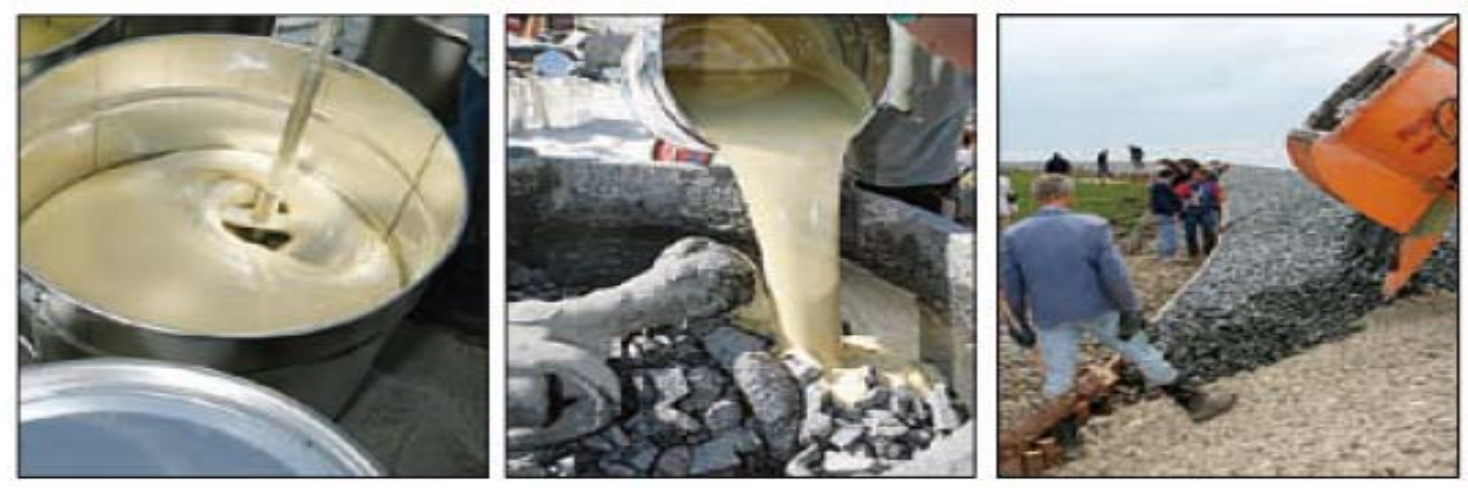

Figure 2. Les étapes de mise en ouvre d'un enrochement lié par Elastocoast ${ }^{\circledR}$.

\section{Propriété majeure : absorption de l'énergie des vagues}

La structure poreuse de l'ouvrage absorbe une partie de l'énergie hydraulique par frottement dans le volume des pores, ce qui induit les effets suivants :

- Réduction de la hauteur et de la course de la vague.

- Libération plus rapide de la pression exercée par l'eau lorsque la marée redescend.

- Réduction du dimensionnement de l'ouvrage.

- Meilleure résistance au phénomène de surverse.

- Colonisation de la structure cavitaire par la faune et la flore ambiante.

\section{Mise en décharge}

En cas de démolition, les gravats contenant moins de 5\% en volume de polyuréthane, par référence au catalogue européen sur les déchets EWC 070212 (1999), sont soumis à l'EWC 170107 ou 170504 (Mélange de béton, de briques, de carrelage, de céramique : recyclage comme matériaux du bâtiment possible).

\section{Résultats de quelques essais}

\subsection{Jet de rive}

Plusieurs méthodes de calcul qui estiment la hauteur du jet de rive, utilisent un facteur de réduction pour tenir compte de la rugosité du revêtement $\gamma_{\mathrm{f}}$. Pour la conception d'un PBA-REVETMENT la valeur choisie devrait être égale à celle d'un revêtement bitumineux poreux, à savoir $\gamma_{\mathrm{f}}=0.9$. Cette valeur est assez conservative et en pratique une meilleure valeur devrait être trouvée. A partir d'essais sur des prototypes des facteurs de 0,60 à 0,65 ont été observés.

\subsection{Résistance en milieu marin - Corrélation d'Arrhenius}

La résistance en milieu marin a été validée par la méthode dite Corrélation D’Arrhenius (voir figure 3) qui permet une extrapolation à des températures comprises entre 20 et $30^{\circ} \mathrm{C}$. 


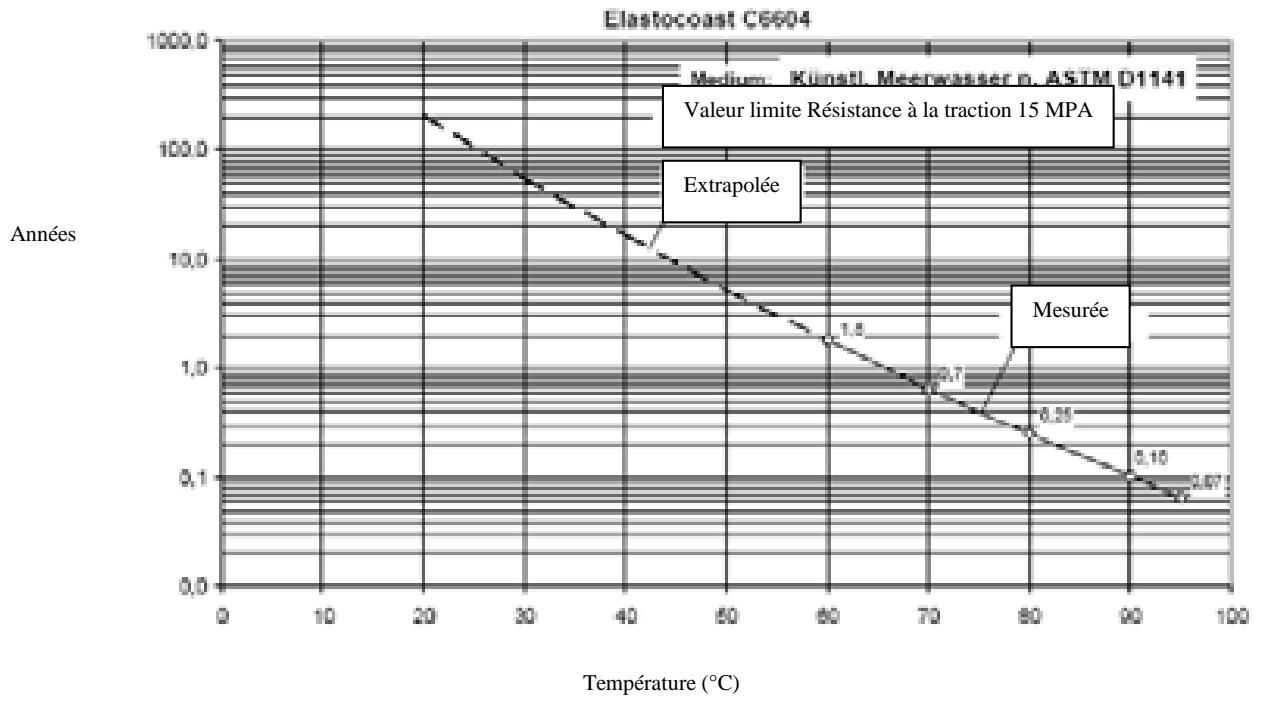

Figure 3. Résistance dans le temps en fonction de la température Corrélation d'Arrhenius.

\subsection{Rigidité}

Elle dépend de la nature, de la granulométrie de l'agrégat et de la température, voir figure 4 (BIJLSMA, 2008).

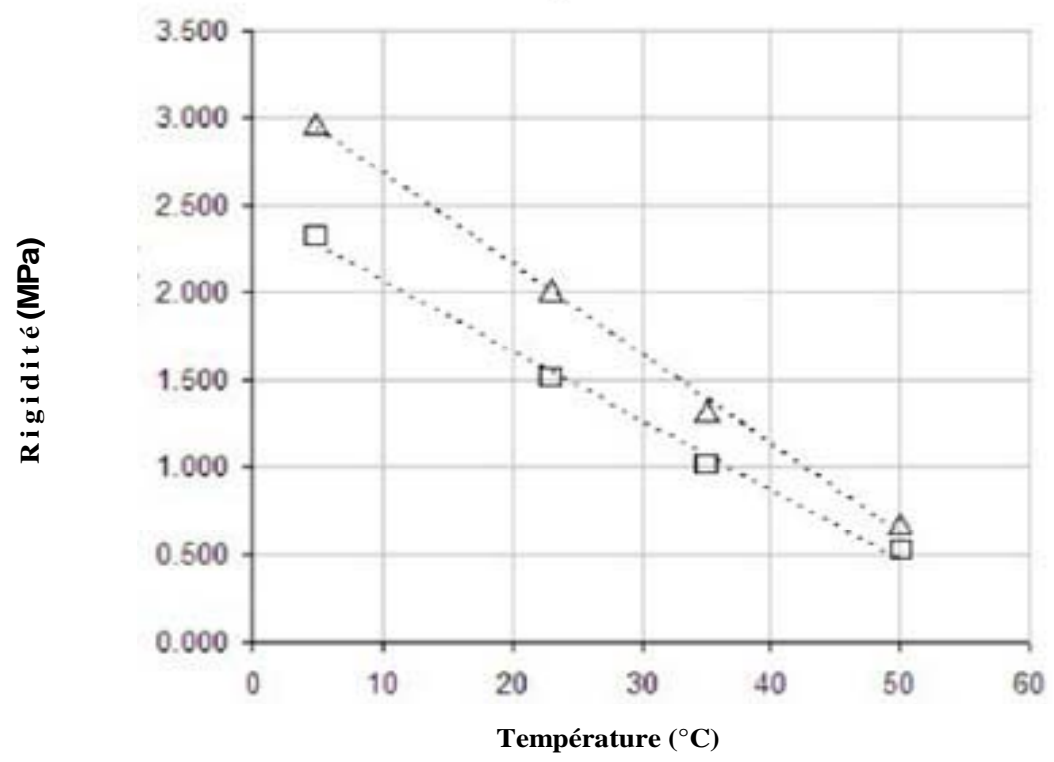

Figure 4. Rigidité en fonction de la température et de la granularité.

Pour les calculs la valeur conservative de $\mathrm{E}=4,000 \mathrm{MPa}$ peut être utilisée (BIJLSMA, 2008). 


\section{XI ${ }^{\text {èmes }}$ Journées Nationales Génie Côtier - Génie Civil \\ Les Sables d'Olonne, 22-25 juin 2010}

\section{$6.4 \underline{\text { Rupture à la flexion }}$}

Elle dépend fortement de la granulométrie, de la granularité, du type d'agrégats et de la température, voir tableau 1.

Tableau 1.

\begin{tabular}{llll}
\hline Composition & $\begin{array}{l}\text { Flexion } \\
\text { Moyenne }\end{array}$ & Ecart-type & $\begin{array}{l}\text { Module de } \\
\text { référence }\end{array}$ \\
\hline Calcaire $8 / 11 \mathrm{~mm}, 3 \%$ en vol $\mathrm{PU}$ & $2,51 \mathrm{MPa}$ & $0,28 \mathrm{MPa}$ & $1,0 \mathrm{MPa}$ \\
Calcaire $8 / 11 \mathrm{~mm}, 3 \%$ en vol $\mathrm{PU}$ & $2,85 \mathrm{MPa}$ & $0,64 \mathrm{MPa}$ & $1,0 \mathrm{MPa}$ \\
\hline
\end{tabular}

6.5 Résistance au phénomène de surverse

Le PBA-REVETMENT a été testé par le "Projectbureau Zeeweringen, The Netherlands" dans des conditions maximales dues à la capacité de l'équipement à $125 \mathrm{l} / \mathrm{s} / \mathrm{m}$ sans aucun dommage significatif, voir figure 5 .

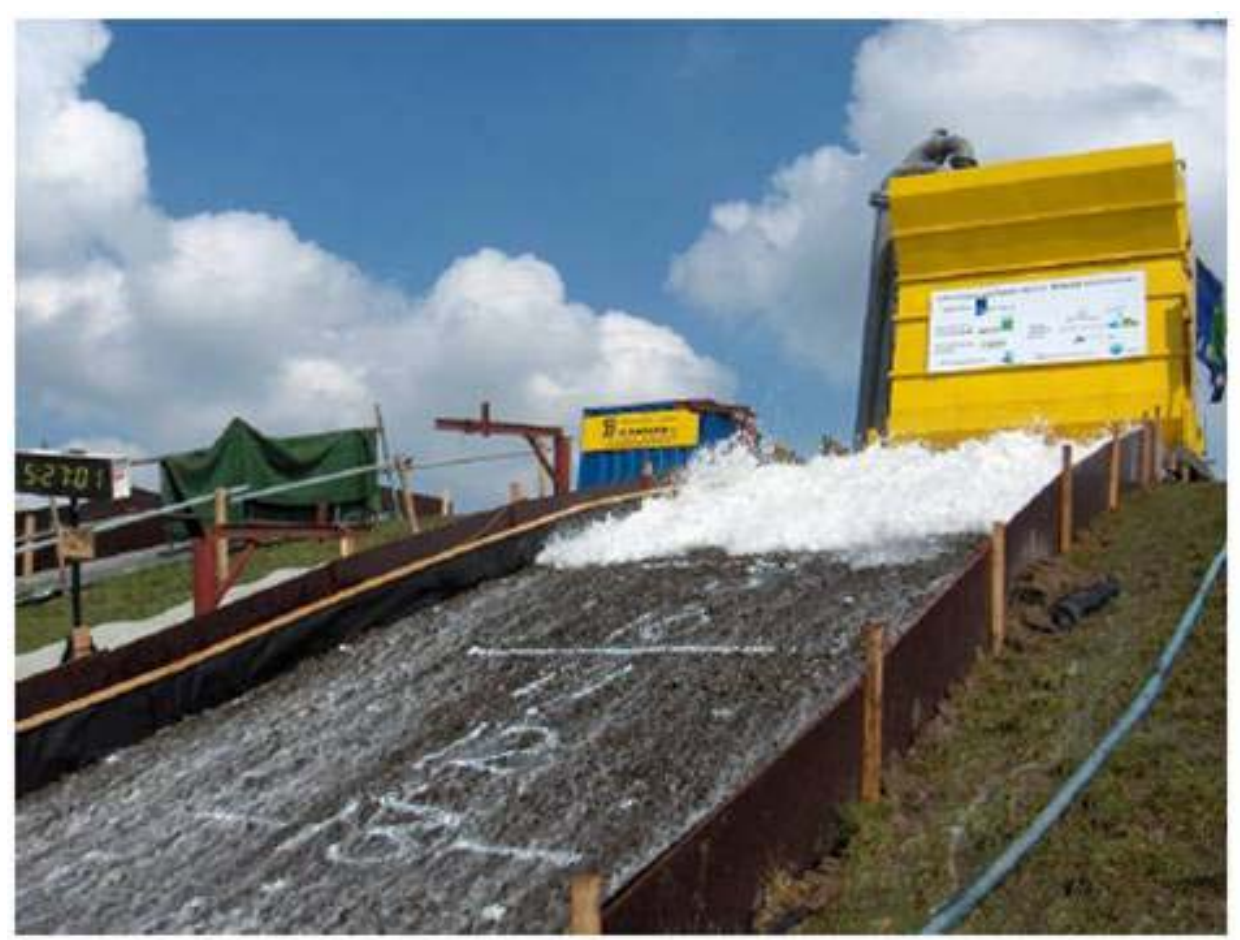

Figure 5. Test de surverse

\section{Exemple de dimensionnement}

La figure 6 illustre un exemple de dimensionnement de l'épaisseur de revêtement pour différentes pentes du talus, en fonction de la hauteur de vague. 


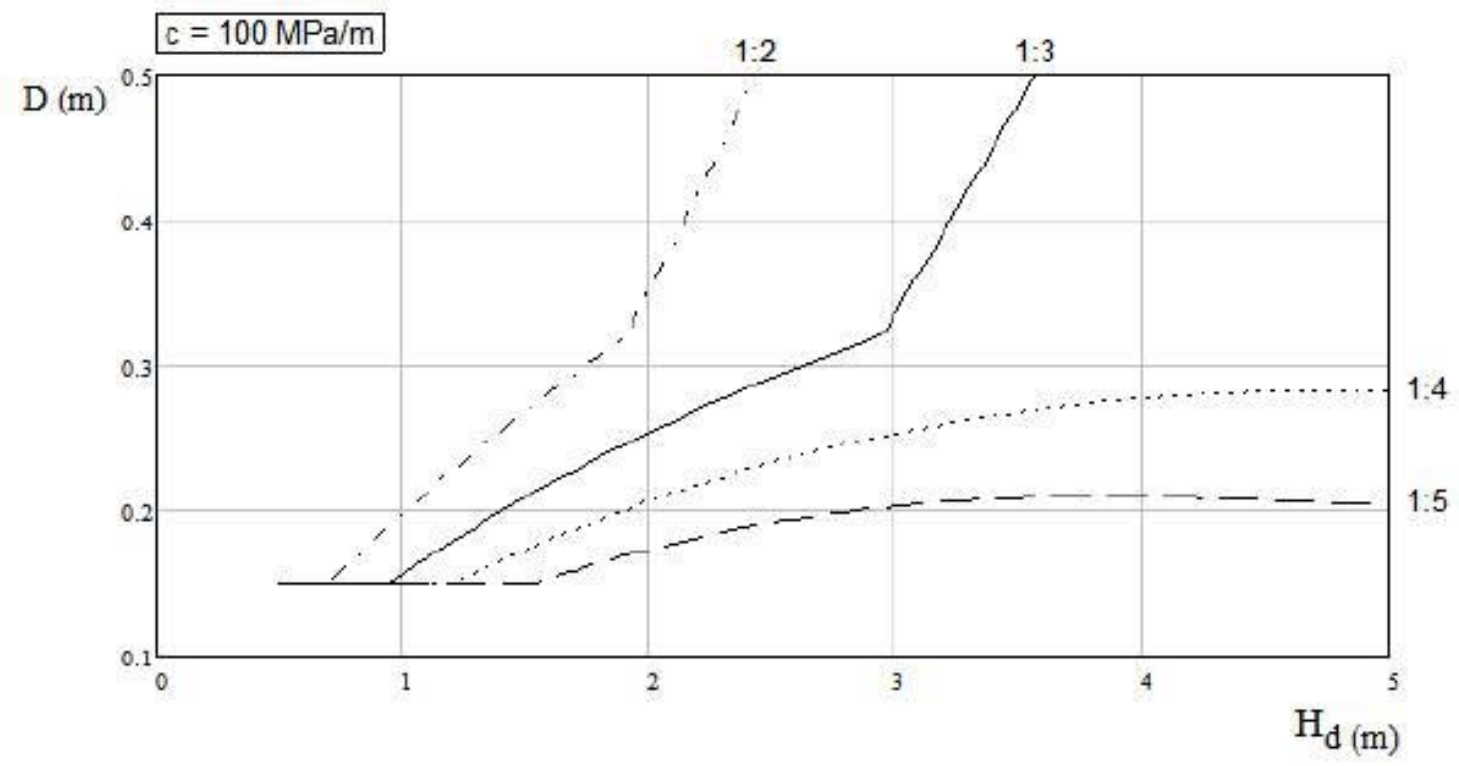

Figure 6. Epaisseur de revêtement (D) en fonction de la hauteur de vague (H) directement sur sable et géotextile ou sur une couche granulaire filtrante, $E=4.000 \mathrm{MPa}, \sigma_{\max }=1,000 \mathrm{MPa}$.

\section{Quelques réalisations}

Des exemples de réalisations sont présentés dans le tableau 2.

Tableau 2. Exemples de réalisations.

\begin{tabular}{|c|c|c|c|c|}
\hline Pays & Lieux & Année & Types & Risques \\
\hline Allemagne & Hamburger-Hallig Mer du Nord $120 \mathrm{~m}^{2}$ & 2004 & Brise lames & Inondations, surcotes \\
\hline Allemagne & Sylt Mer du Nord $-270 \mathrm{~m}^{2}$ & 2005 & Brise lames & Inondations \\
\hline \multirow[t]{2}{*}{ Allemagne } & Gröde Mer du Nord - $5000 \mathrm{~m}^{2}$ & 2006 & Brise lames & Inondations, surcotes \\
\hline & & 2007 & Rénovation & \\
\hline Allemagne & Sylt-Munkmarsch Mer du Nord-1500 $\mathrm{m}^{2}$ & 2007 & Brise lames & Inondations \\
\hline Pays-Bas & Zuidbout $-510 \mathrm{~m}^{2}$ & 2007 & Epi & \\
\hline Pays-Bas & Petten $-500 \mathrm{~m}^{2}$ & 2007 & Digue & Inondations \\
\hline Pays-Bas & Bathpolder $-12000 \mathrm{~m}^{2}$ & 2009 & Digue & Inondation \\
\hline Canada & Detroit River $-510 \mathrm{~m}^{2}$ & 2007 & Protection berge & Batillage, gel \\
\hline Royaume-Uni & Holland on Sea $-300 m^{2}$ & 2008 & Protection & Erosion \\
\hline France & Canal Tancarville (P. du Havre) $60 \mathrm{~m}^{2}$ & 2008 & Protection berge & Batillage \\
\hline France & Musoir écluse de Bollène (CNR) - $60 \mathrm{~m}^{2}$ & 2009 & Musoir & Batillage \\
\hline France & Port de Rouen $-80 \mathrm{~m}^{2}$ & 2009 & Protection berge & Batillage \\
\hline France & La Bouille (Départ76) - $80 \mathrm{~m}^{2}$ & 2009 & Protection berge & Batillage \\
\hline France & Grand Port Maritime Bordeaux - $360 \mathrm{~m}^{2}$ & 2009 & Protection berge & Houle \\
\hline
\end{tabular}




\section{XI $I^{\text {èes }}$ Journées Nationales Génie Côtier - Génie Civil}

Les Sables d'Olonne, 22-25 juin 2010

\section{Référencements}

Les premiers référencements sont les suivants :

- Fiche Elastocoast ${ }^{\circledR}$ dans le Club Ouvrages maritimes du CETMEF, octobre 2009;

- Ouvrage Aménagements des berges des voies navigables - Retour d'expériences, Edition CETMEF (GALIANA et al., 2009).

\section{Exemples illustrés}

La figure 7 illustre 6 exemples d'application de la technologie Elastocoast ${ }^{\circledR}$ (BASF, 2008 ; DE GARDER, 2009)
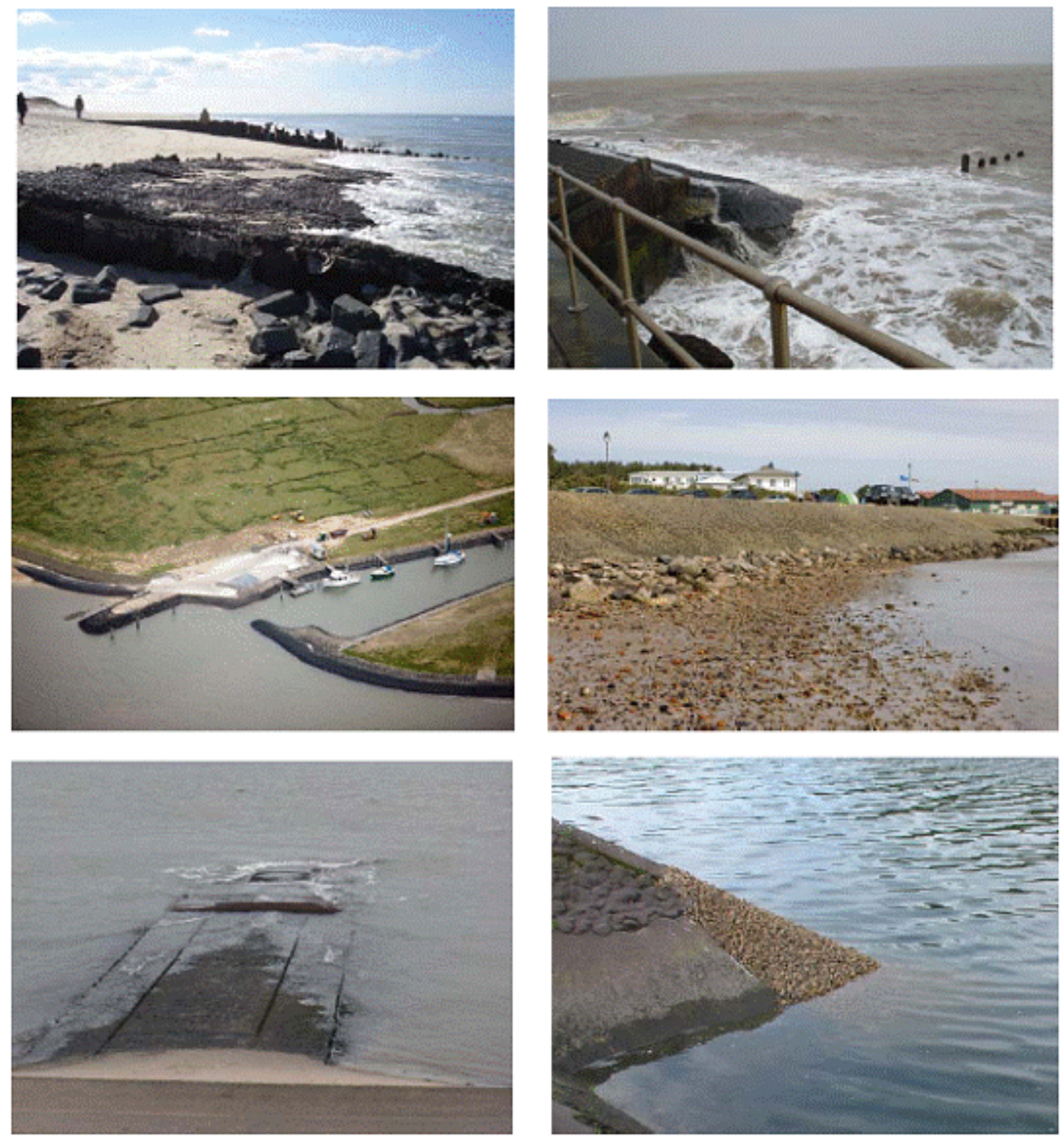

Figure 7. Exemples d'application de la technologie Elastocoast ${ }^{\circledR}$. 
Thème 5 -Ouvrages portuaires, côtiers et offshore

\section{Références bibliographiques}

BASF (2008). Elastocoast - An innovative technology in Coastal Protection... ...today and tomorrow: Information, References and Documents. 112 p. Document disponible en ligne sur : URL http://www.elastogran.de/ftp/Elastocoast_handbuch_en.pdf BIJLSMA E. (2008). Elastocoast pilots in the Netherlands: Stormseason 2007/2008. Delft, The Netherlands, Delft University of Technology, Master's Thesis, $93 \mathrm{p}$. DE GARDER P. (2009). Elastocoast ${ }^{\circledR}$ - Process innovateur dans la construction d'ouvrages en enrochements dans le cadre de la protection du littoral maritime et fluvial. Conférence Méditerranéenne Côtière et Maritime, Hammamet, Tunisie, pp 331-334. doi:10.5150/cmcm.2009.081-2

GALIANA M., LE BRIS D., DUBOIS D., DE BRUYN B. (2009). Aménagement des berges des voies navigables - Retour d'expériences. Edition CETMEF, $95 \mathrm{p}$. 